\title{
Hyperspectral Remote Sensing Data Mining Using Multiple Classifiers Combination
}

\author{
Xing-Ping Wen ${ }^{1}$, Xiao-Feng Yang ${ }^{1}$ and Guang-Dao $\mathrm{Hu}^{2}$ \\ ${ }^{1}$ Faculty of Land Resource Engineering, Kunming University of science and technology, \\ 2Institute of Mathematic Geology and Remote Sensing Geology, \\ China University of Geosciences (Wuhan)
}

China

\section{Introduction}

Since the advent of remote sensing in the second half of 20th century, nowadays there have been great changes in theory and technology. The advent of hyperspectral was one of the most significant breakthroughs in remote sensing. Hyperspectral remote sensing has higher spectral resolution as the same time retain higher spatial resolution, so its capability of distinguishing the different and describing the same ground objects in details enhanced greatly. It acquires image in a large number (typically over 40), narrow (typically 10 to 20 $\mathrm{nm}$ in width) and contiguous spectral bands to enable the extraction of reflectance spectra at a pixel scale, so it can produce data with sufficient resolution for the direct identification of those materials with diagnostic spectral features (Goetz et al., 1985). The objective of hyperspectral remote sensing is to measure quantitatively the components of the Earth System from calibrated spectra acquired as images for scientific research and applications (Vane \& Goetz, 1988). The rationale behind this technology for geological applications is that mineral species have diagnostic absorption features from 20 to $40 \mathrm{~nm}$ wide in electromagnetic wavelength ranges which is larger than hyperspectral spectral resolution (van der Meer \& Bakker, 1997). Goetz demonstrated firstly that direct identification of carbonates and hydroxyl-bearing minerals is possible by remote measurement from Earth orbit (Goetz et al., 1982).

There are two main categories of extracting information method from hyperspectral remote sensing image: based on feature space and based on spectral space. Many statistics-based classification methods based on feature space have been successfully applied to multispectral remote sensing data in the past years (Pal \& Mather, 2003, Wen et al., 2008b). However, they are not effective for hyperspectral remote sensing data. The problem is caused by curse of dimensionality and Hughes phenomenon (Hsu, 2007), which refer to the fact that the sample size required for training a specific classifier grows exponentially with the number of spectral bands. Usually simple but sometimes effective ways to overcome this problem is to increase sample numbers or to reduce the dimensionality of hyperspectral remote sensing data. The former needs a lot of sample numbers, so it will cost many human and material resources; the latter will lead to some useful information lost. The Matched Filtering methods based on spectral space are successfully used in hyperspectral data. These 
methods based on the hypothesis that all spectra have been calibrated to apparent reflectance and the dark current of sensor and path radiation is removed. It is only the ideal state for these effects are hard to remove successfully, especially in low reflectance object, so this will lead to error using Matched Filtering.

In order to obtain the best classification performance in pattern recognition, the data set should be classified using different methods, and then choose the best classification result as the final conclusion. With the complexity of pattern recognition increased and novel algorithm developed, researchers find that although different classifiers have different classification performance, their misclassification set are not consistent with each other. That is, some sample misclassified by one classifier may be recognized by another classifier. Different classifiers are complementary to each other. If only the best performance classifier is chose, some valuable information from other classifiers may be ignored. In order to solve this problem, multiple classifiers combination was put forward. This chapter proposed the methods to improve the hyperspectral remote sensing classification accuracy using multiple classifiers combination. The method included two parts: one is to improve the performance of the single classifier, and the other is to combine multiple classifiers. In the former, the chapter investigated the methods of atmospheric correction and extracting the purer endmember, and a novel endmember extracting method combining multi-segmentation and geology map was proposed. In the latter, combining multiple classifiers based on decision tree was proposed.

The study area is located in southwest of China, and the composition of the deposits is mainly dioritic porphyrite. Firstly, the image was atmospherically corrected before processing, and a endmember was extracted by PPI algorithm from the intersection area of multi-segmentation and geology map; Secondly, dioritic porphyrite area was extracted from hyperspectral remote sensing image by Spectral Angle Mapper (SAM), Multi Range Spectral Feature Fitting (Multi Range SFF) and Mixture Tuned Matched Filtering (MTMF) using the extracted endmember respectively. At last, final classification results was outputted by combining three classification results using Classification and Regression Trees (CART). Comparing all classification results and geology map, it is concluded that combining multiple classifiers has the best classification performance and Multi Range SFF has the better capable of pixel un-mixing than SAM and MTMF.

\section{Study area and hyperspectral remote sensing data}

The study area is located at Pulang porphyry copper and gold deposit in Shangri-La of Yunnan province in southwest of China (fig. 1). The longitude and latitude scope are between $26^{\circ} 54^{\prime} \mathrm{N}-28^{\circ} 43^{\prime} \mathrm{N}$ and $99^{\circ} 37^{\prime} \mathrm{E}-100^{\circ} 10^{\prime} \mathrm{E}$. Its ore-bearing lithology mainly contains dioritic porphyrite. The hyperspectral remote sensing employed in this paper was acquired by Hyperion sensor on board EO-1 satellite in December 2, 2003. The EO-1 satellite was launched on November 21, 2000 as part of a one-year technology validation/demonstration mission by NASA. The original EO-1 Mission was successfully completed in November 2001. As the end of the Mission approached, an agreement was reached between NASA and the USGS to allow continuation of the EO-1 Program as an Extended Mission based on the remote sensing research and scientific communities interest and willingness to assist in funding continued operations. The three primary instruments on the EO-1 spacecraft are the Advanced Land Imager (ALI), the Hyperion, and the Linear Etalon Imaging Spectrometer Array (LEISA) Atmospheric Corrector (LAC). The Hyperion covers 400-2500 nm with 242 
spectral bands over a $7.5 \mathrm{~km}$ wide swath at approximately 10nm (sampling interval) spectral resolution and $30 \mathrm{~m}$ spatial resolution on a $705 \mathrm{~km}$ orbit.

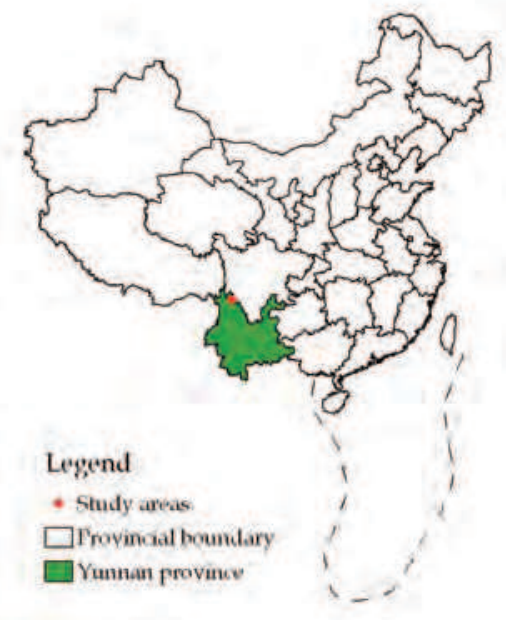

Fig. 1. China provincial boundaries and study area

\section{Atmospheric correction of hyperspectral remote sensing data}

Atmospheric effects play a dominant role in the optical part of the electromagnetic spectrum. It generates a variety effects upon the satellite image that must subsequently be accounted for through atmospheric corrections. To have lasting quantitative value, remotely sensed data must be calibrated to physical units of reflectance (Smith \& Milton, 1999). In order to remove accurately atmospheric absorption and scattering effects, atmospheric correction algorithms have evolved from the earlier empirical line method and flat field method to more recent methods based on rigorous radiative transfer modeling. MODTRAN, the Air Force Research Laboratory/Geophysics Directorate moderate spectral resolution (2 $\mathrm{cm}^{-1}$ ) background radiance and transmittance model, has been widely used to analyze hyperspectral data to its computational speed and its ability to model molecular and aerosol/cloud emissive and scattered radiance contributions as well as the atmospheric attenuation (Berk et al., 1998). It accurately and efficiently calculates the scattering and absorption signatures of realistic molecular, aerosol and cloudy environments in the lower and middle atmosphere. There are several MODTRAN-based atmospheric correction software packages, such as FLAASH (Fast Line-of-sight Atmospheric Analysis of Spectral Hyper-cubes) (Anderson et al., 1999), ATCOR (Atmospheric and Topographic Correction) (Richter, 1996) and others. ATCOR was originally developed by Richter at DLR, the German Aerospace Centre. The algorithm is a fast atmospheric correction for imagery of medium and high spatial resolution satellite sensors. There are two ATCOR models available, one for satellite imagery, the other one for airborne imagery. For historic reasons, the satellite codes are called ATCOR-2 (Richter, 1996) and ATCOR-3 (Richter, 1998). ATCOR-2 is used for flat terrain and ATCOR-3 is for Mountainous Terrain. ATCOR3 includes all of the capabilities of ATCOR2 and can be integrated with a DEM for atmospheric correction of images depicting 
rugged terrain. FLAASH (Fast Line-of-sight Atmospheric Analysis of Spectral Hypercubes) is being developed by the Air Force Research Laboratory, Hanscom AFB, and Spectral Sciences, Inc., to support current and planned SWIR/visible/UV hyperspectral and multispectral sensors, typically in image format.

In order to compare correction effect of different algorithms, in this chapter, ACTOR-2, ACTOR-3 and FLAASH were used to remove atmospheric effects from the hyperspectral data. Fig. 3 is the reflectance of the same pixel from Hyperion remote sensing data corrected atmospherically by ACTOR-2, ACTOR-3 and FLAASH. Due to complex terrain of study area, where maximum elevation difference is greater than $1200 \mathrm{KM}$ (fig.2), the correction effect of ACTOR-3 is not efficient. Some reasons may be originated from the rough DEM data and geometric correction error. As a result, the Hyperion remote sensing data calibrated to apparent reflectance using the FLAASH was selected to extract information.
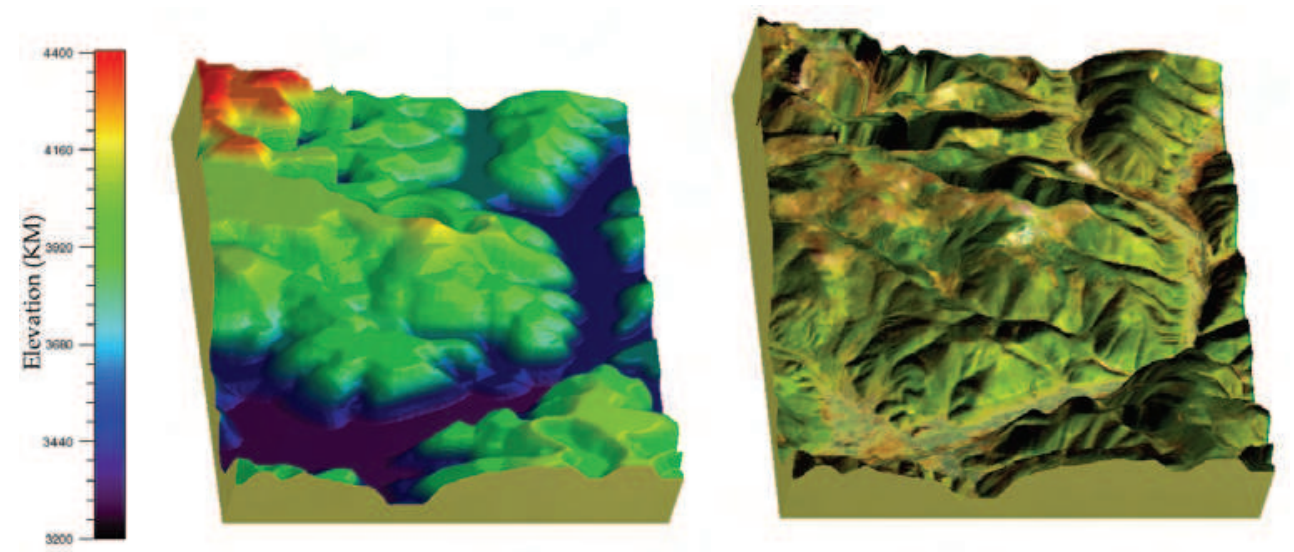

Fig. 2. Digital elevation model ( DEM) of study area (left) and 3D surface view image using hyperion remote sensing data

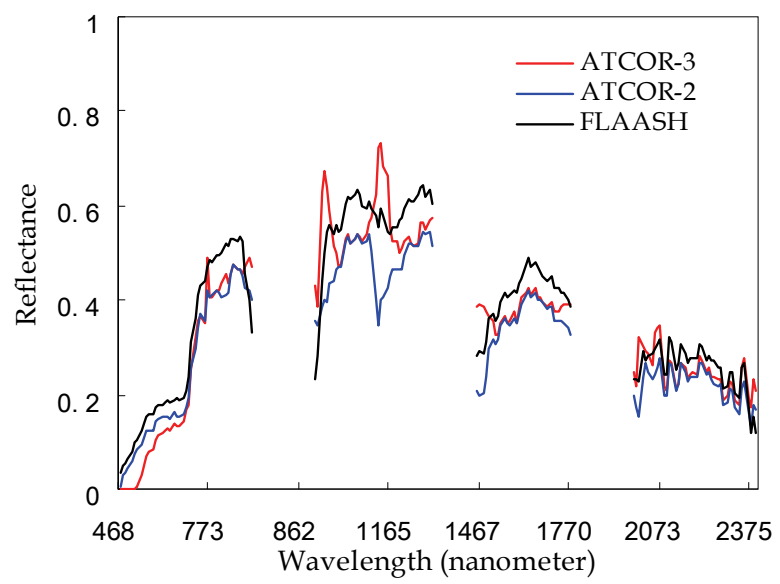

Fig. 3. The reflectance of the same pixel using different atmospheric correction model 


\section{Methodology}

\subsection{Endmember extraction}

Mineral mixtures and mixtures with vegetation in an individual pixel can be separated if the components have unique spectral features (endmember). Many researchers have used an ndimensional analysis approach to determine key endmember and map their distribution and abundance (Boardman, 1993). A variety of methods have been proposed to find endmembers in multispectral and hyperspectral images. Iterated Constrained Endmembers (ICE) is an automated statistical approach to identifying endmembers from hyperspectral images (Berman et al., 2004). Winter proposed to find a unique set of purest pixels based upon the geometry of convex sets (Winter, 1999). Probably the most widely used algorithm is Pixel Purity Index (PPI) (Boardman et al., 1995). PPI usually used to find the most spectrally pure (extreme) pixels in multi-spectral and hyperspectral images. (Kruse, 2005) used multi-resolution segmentation (Baatz \& Schäpe, 2000) to separate adjacent regions in an image, then using PPI to extract endmember. Multi-resolution segmentation is a bottom up region-merging technique starting with one-pixel objects. In numerous subsequent steps, smaller image objects are merged into bigger ones. It can slice the image into a network of homogeneous image regions at any chosen resolution, even when the regions themselves are characterized by a certain texture or noise. In this chapter, multi-resolution segmentation regions in hyperspectral remote sensing image were intersected with the dioritic porphyrite area in geologic map (fig. 4), so the overlay areas contain homogeneous dioritic porphyrite area. Then, one overlap area was selected to extract dioritic porphyrite endmember using PPI.
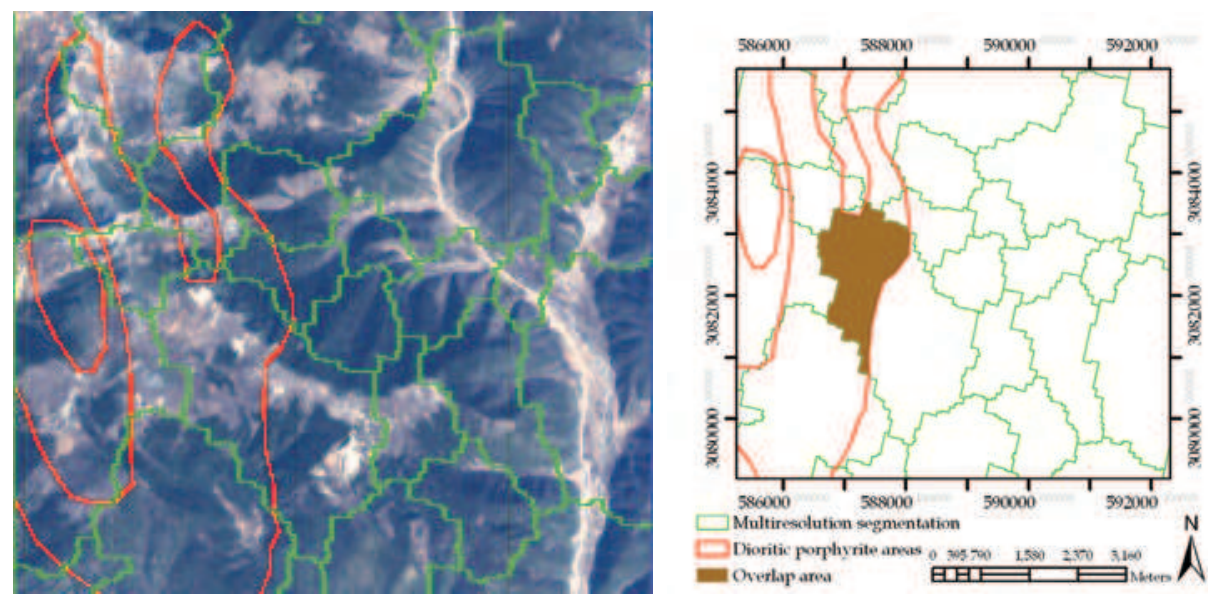

Fig. 4. The hyperspectral remote sensing image (left) and the overlap area where multiresolution segmentation intersects dioritic porphyrite area in geologic map (right)

\subsection{Matched filtering methods}

Each pixel in the image is a mixture of responses from multiple materials and nearly no pure pixels are present in the image due to large sampling distance $(30 \mathrm{~m})$. Matched Filtering (MF) method can find the abundances of user-defined endmembers using a partial unmixing. There are many MF methods. SAM, MTMF and Multi Range SFF are widely used to 
extract specific materials based on matches to library or image endmember spectra and does not require knowledge of all the endmembers within an image scene.

\subsubsection{SAM algorithm}

SAM is a physically-based spectral classification that uses an n-dimension angle to match pixels to endmember spectra. The algorithm determines the spectral similarity between two spectra by calculating the angle between the spectra, treating them as vectors in a space with dimensionality equal to the number of bands (Kruse et al., 1993). The endmember spectra can be either laboratory or field spectra or extracted from the image. This method assumes that the data have been reduce to apparent reflectance, with all dark current and path radiance biases removed. This technique, when used on calibrated reflectance data, is relatively insensitive to illumination and albedo effects. SAM algorithm has successfully used in geological mapping based on remote sensing data (Baugh et al., 1998, Wen et al., $2007 \mathrm{~b}$ ). It computes the "spectral angle" between pixel spectra and the endmember spectra. Smaller angles represent closer matches to the endmember spectra. The result is a rule image that indicates the radian of the spectral angle (fig. 6(a)). The radian of the spectral angle calculated by applying the following equation:

$$
\alpha=\cos ^{-1}\left(\frac{\sum_{i=1}^{n b} t_{i} r_{i}}{\left(\sum_{i=1}^{n b} t_{i}^{2}\right)^{1 / 2}\left(\sum_{i=1}^{n b} r_{i}^{2}\right)^{1 / 2}}\right)
$$

Where $\mathrm{nb}=$ the number of bands, $t_{i}=$ pixel spectrum, $r_{i}=$ endmember spectrum.

\subsubsection{Multi range SFF}

$\mathrm{SFF}$ is an absorption-feature-based methodology. The endmember spectra are scaled to match the image spectra after the continuum is removed from both data sets. Multi Range SFF uses Continuum Removal (CR) to normalize reflectance spectra so individual absorption features from a common baseline can be compared. An apparent continuum in a reflectance spectrum is modeled as a mathematical function that is used to isolate a particular absorption feature for analysis, and this continuum should be removed by dividing it into the reflectance spectrum for each pixel in the image (Clark \& Roush, 1984):

$$
S_{C R}=S / C
$$

Where: $S_{C R}=$ Continuum removed spectra, $S=$ Original spectra, $C=$ Continuum curve.

The continuum curve is a convex hull fit over the top of a spectrum using straight-line segments that connect local spectra maxima, so the first and last spectral data values are on the hull, therefore, the first and last bands in the output continuum removed data are equal to 1.0. Absorption feature analysis using CR has been shown to enhance the differences in shape between the absorption features of interest (Kokaly \& Clark, 1999). Multi Range SFF compares the CR of image spectra to the CR of endmember spectra at each wavelength using a least-squares technique. Scale image and RMS image are output for each 
endmember spectrum. The scale image is a measure of absorption feature depth, which is related to material abundance. The brighter pixels in the scale image indicate a better match to the endmember material in those pixels. The RMS error is calculated for each endmember spectrum, and dark pixels in the RMS image indicate a low error. Finally, the fit image with the higher abundance and a low error value which was calculated by dividing RMS image into the Scale image is output for the endmember spectrum (fig. 6(b)).

\subsubsection{MTMF}

MTMF combines the best parts of the Linear Spectral Mixing model and the statistical Matched Filter model while avoiding the drawbacks of each parent method (Boardman, 1998). It is a useful Matched Filter method without knowing all the possible endmembers in a landscape especially in case of subtle, sub-pixel occurrences. Firstly, pixel spectra and endmember spectra require a minimum noise fraction (MNF) (Green et al., 1988, Boardman, 1993) transformation. MNF reduces and separates an image into its most dimensional and non-noisy components. Once the data is in a less noisy form, it can then be compared to endmember through MTMF processes to determine composition. Its results appear as two gray-scale images for a selected endmember spectrum, one scale image estimate the relative degree of match to the endmember spectrum and the approximate sub-pixel abundance, the other is an infeasibility image used to reduce the number of false positives. Better matched spectra from combination image which was calculated by dividing infeasibility image into the scale image will have a higher abundance and a low infeasibility value (fig. 6(c)).

\subsection{Multiple classifiers combination}

Due to the complexity of land cover, statistical distribution characteristics of the remote sensing data vary in time and space, so the classification accuracy of different classifiers are obviously different. Experiments reported by the authors and other researchers have clearly shown that the superiority of one algorithm over another cannot be claimed for remotesensing image classification (Giacinto et al., 2000). Multiple classifiers combination will improve the classification accuracy by using different classifiers complementarities.

Multiple classifiers systems are special cases of approaches that integrate several datadriven models for the same problem. Its key goal is to obtain a better composite global model, with more accurate and reliable estimates or decisions (Ghosh, 2002). The theory of multi-classifier systems can be traced back at least as far as 1965 (Nilsson, 1965). Previous multiple classifiers combination algorithm include the voting (Lam \& Suen, 1994), Bayes rule (Xu et al., 1992), Dempster-Shafer theory (Mandler \& Schuermann, 1988), decision tree and other methods. Based on classifier outputs, the multiple classifiers combination methods can be classified into three different levels: abstract level, ranked list of classes, and measurements (Suen \& Lam, 2000, Xu et al., 1992). Based on manipulating training samples, they can be classified into two approaches: boosting (Freund \& Schapire, 1996) and bagging (Breiman, 1996). Ideally, the combination should take advantage of the merit of the individual classifiers, avoid their weaknesses and improve classification accuracy.

Multiple classifiers combination was successfully used in remotely sensed data classification. However, the standard multiple classifiers combination methods were seldom used in remotely sensed data classification. (Wen et al., 2007a) combined three MF results 
using adjusting threshold method, and demonstrated the result was better than only one MF result. However, it is hard to get the effective split value only using adjusting the threshold by hand. In this chapter, CART (Brieman et al., 1984, Wen et al., 2008a) was used to extract the rock body information from the hyperspectral remote sensing image by combine three MF results. CART method was suggested by Breiman et al. It partitions the data into two subsets of records with similar values for the target attribute so that the records within each subset are more homogeneous than in the previous subset. It is a recursive process: each of those two subsets is then split again, and the process repeats until the homogeneity criterion is reached or until some other stopping criterion is satisfied. It allows unequal misclassification costs to be considered in the tree growing process. The decision trees produced by CART are strictly binary, containing exactly two branches for each decision node. The CART algorithm grows the tree by conducting for each decision node, an exhaustive search of all available variables and all possible splitting values, selecting the optimal split according to the certain criteria.

\section{Conclusion}

The decision tree constructed by CART algorithm is shown in fig. 5. "Y" in the rectangle means the pixel contains dioritic porphyrite. On the contrary, " $\mathrm{N}$ " in the rectangle means the pixel contains no dioritic porphyrite. The value near the rectangle is confidence. From fig. 5, when the value of Multi Range SFF and MTMF rule image are greater than the certain threshold, it concludes the pixel contains dioritic porphyrite. They are coincided with their physical meaning for that the higher value of Multi Range SFF and MTMF rule image is

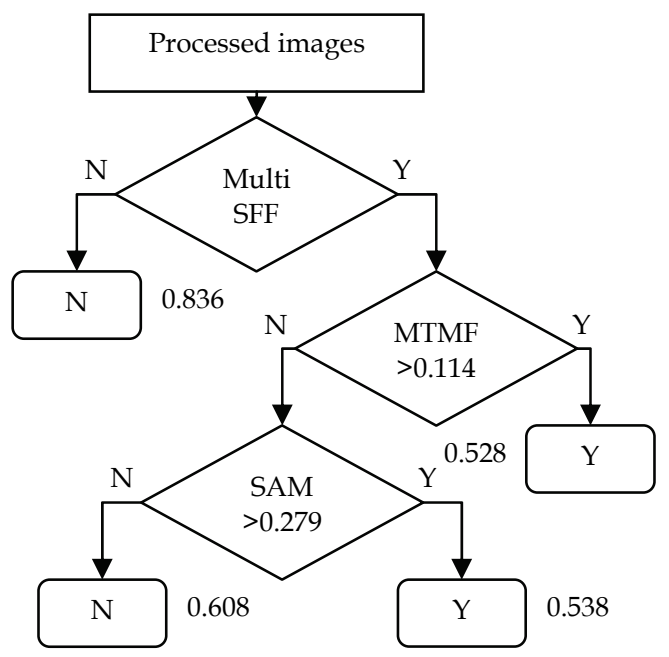

Fig. 5. Decision tree constructed by CART algorithm

related to higher material abundance. However, when the value of SAM rule image is greater than the certain threshold, it concludes the pixel contains dioritic porphyrite. It is inconsistent with its physical meaning for the smaller value of SAM rule image represents closer matches to the endmember spectra. The reason maybe is that SAM only compares the 
shape of spectra, so its ability of pixel un-mixing is limited. Comparing three methods, the decision tree also show that Multi Range SFF has great pixel un-mixing ability; followed by MTMF; SAM is the least. Most alteration minerals have diagnostic spectral absorption features in the short wave and mid-infrared. Multi Range SFF compares enhanced spectra absorption features, so it is effective. Three MF results and combining three MF results using CART were used to extract dioritic porphyrite respectively. The rule images generated by different methods are shown in fig. 6. Comparing four rule images, the combination rule image is better than others significantly. Using dioritic porphyrite areas in geologic map as ground truth, the classification accuracy are calculated and results are shown in table 1 . As is shown from table 1, multiple classifier combination has the best classification performance, and it generates the highest overall accuracy and hit/false alarm. The final Dioritic porphyrite map of Pulang using combining multiple classifiers is shown in fig. 7 . Comparing with the field data and geology map, it concludes that this method is effective.

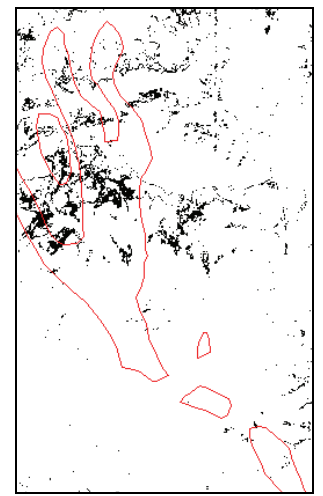

(a) SAM

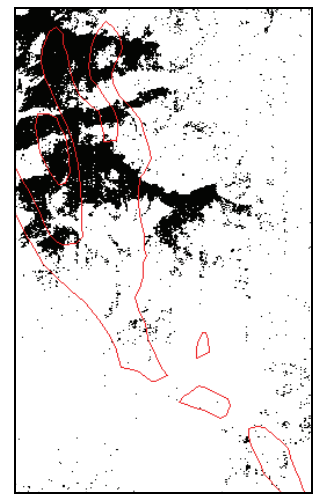

(b) Multi Range SFF

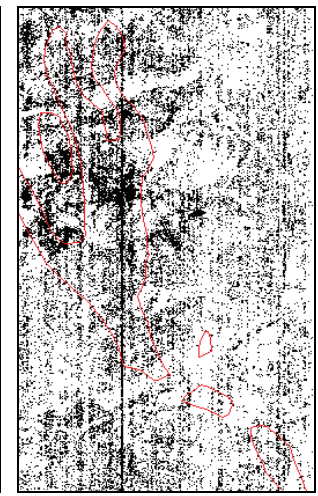

(c) MTMF

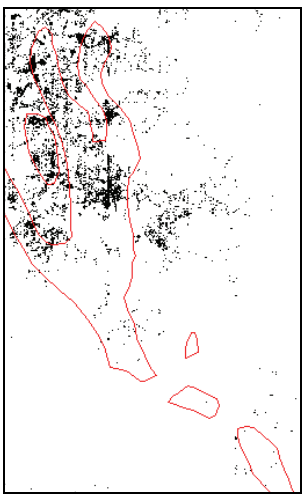

(d) Combination

Fig. 6. Rule images generated by different methods

\begin{tabular}{|c|c|c|c|c|}
\hline Method & Hit (pixels) & False alarm (pixels) & Overall accuracy & Hit/false alarm \\
\hline SAM & 1858 & 2972 & 0.769 & 0.625 \\
\hline Multi SFF & 6971 & 9137 & 0.756 & 0.763 \\
\hline MTMF & 6809 & 13702 & 0.698 & 0.497 \\
\hline Combination & 2524 & 2239 & 0.786 & 1.127 \\
\hline
\end{tabular}

Table 1. The classification result using different method

SAM is a physically-based spectral classification that determines the spectral similarity. Multi Range SFF is an absorption feature based methodology. MTMF, a special type of spectral mixture analysis, is based on well-known signal processing methodologies. Combining of three matched filtering method can take full advantage of three MF methods, so the classification accuracy is significant improvements than only one approach. 


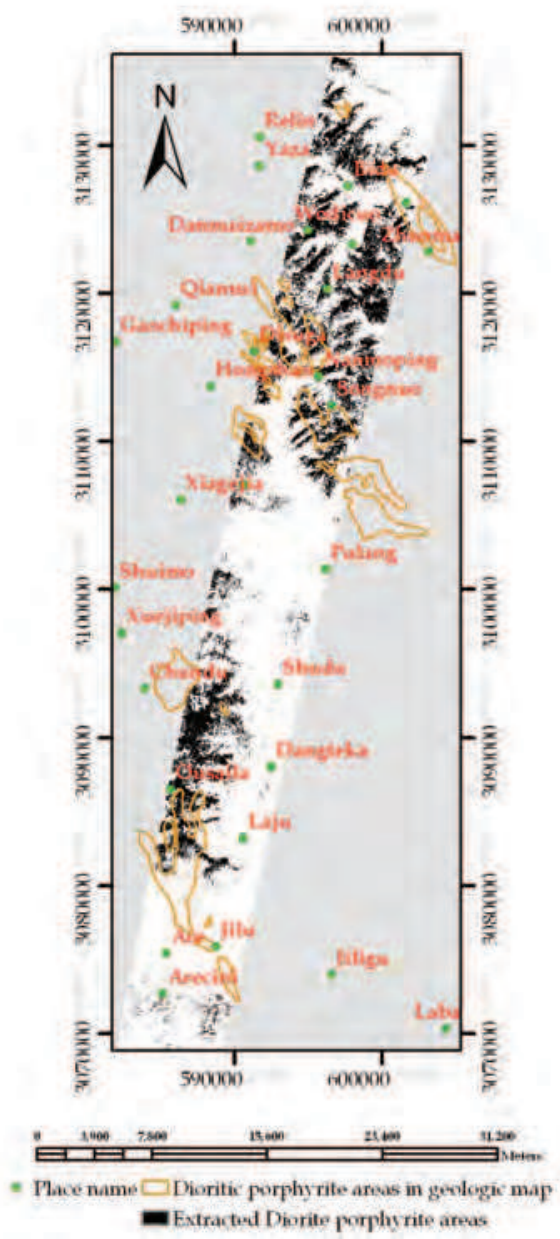

Map Projection: Gauss Kruger, False Easting: 500 kilometer, Central Meridian: $99^{\circ}$.

Fig. 4. Dioritic porphyrite map of Pulang using combining multiple classifiers

\section{Reference}

Anderson, G. P., et al., (1999). FLAASH and MODTRAN4: state-of-the-art atmospheric correction for hyperspectral data. IEEE Proceedings of Aerospace Conference, pp. 177181, Snowmass at Aspen, CO, USA.

Baatz, M. \& Schäpe, A., (2000). Multiresolution segmentation-an optimized approach for high quality multiscale image segmentation. AGIT XIII, pp. 12-23, Wichmann, Heidelberg, Germany.

Baugh, W. M., et al., (1998). Quantitative geochemical mapping of ammonium minerals in the southern Cedar Mountains, Nevada, using the airborne visible/infrared 
imaging spectrometer (AVIRIS). Remote Sensing of Environment, Vol. 65, No. 3, 292308.

Berk, A., et al., (1998). MODTRAN Cloud and Multiple Scattering Upgrades with Application to AVIRIS. Remote Sensing of Environment, Vol. 65, No. 3, 367-375.

Berman, M., et al., (2004). ICE: a statistical approach to identifying endmembers in hyperspectral images. Geoscience and Remote Sensing, IEEE Transactions on, Vol. 42, No. 10, 2085-2095.

Boardman, J. W., (1993). Automated spectral unmixing of AVIRIS data using convex geometry concepts: in Summaries. Fourth JPL Airborne Geoscience Workshop, pp. 1114, Arlington, Virginia, JPL Publication.

Boardman, J. W., (1998). Leveraging the high dimensionality of AVIRIS data for improved sub-pixel target unmixing and rejection of false positives: mixture tuned matched filtering. Summaries of the Seventh Annual JPL Airborne Geoscience Workshop, pp. 5556, Pasadena, CA: NASA Jet Propulsion Laboratory, JPL Publication 97-1.

Boardman, J. W., et al., (1995). Mapping Target Signatures Via Partial Unmixing of Aviris Data. Summaries of the Fifth Annual JPL Airborne Earth Science Workshop, pp. 23-26, Washington, D. C, JPL Publication 95-1.

Breiman, L., (1996). Bagging Predictors. Machine Learning, Vol. 24, No. 2, 123-140.

Brieman, L., et al., (1984). Classification and Regression Trees, Chapman \& Hall/CRC Press. Boca Raton, FL.

Clark, R. N. \& Roush, T. L., (1984). Reflectance spectroscopy-Quantitative analysis techniques for remote sensing applications. Journal of Geophysical Research, Vol. 89, No. B7, 6329-6340.

Freund, Y. \& Schapire, R. E., (1996). Experiments with a new boosting algorithm. Machine Learning: Proceedings of the Thirteenth International Conference, pp. 156, Morgan Kauffman, San Francisco.

Ghosh, J., (2002). Multiclassifier Systems: Back to the Future. Proceedings of the Third International Workshop on Multiple Classifier Systems, pp. 1-15, Cagliari, Italy, Springer.

Giacinto, G., et al., (2000). Combination of neural and statistical algorithms for supervised classification of remote-sensing images. Pattern Recognition Letters, Vol. 21, No. 5, 385-397.

Goetz, A. F. H., et al., (1982). Mineral Identification from Orbit: Initial Results from the Shuttle Multispectral Infrared Radiometer. Science, Vol. 218, No. 4576, 1020-1024.

Goetz, A. F. H., et al., (1985). Imaging Spectrometry for Earth Remote Sensing. Science, Vol. 228, No. 4704, 1147-1153.

Green, A. A., et al., (1988). A transformation for ordering multispectral data in terms of imagequality with implications for noise removal. Geoscience and Remote Sensing, IEEE Transactions on, Vol. 26, No. 1, 65-74.

Hsu, P.-H., (2007). Feature extraction of hyperspectral images using wavelet and matching pursuit. ISPRS Journal of Photogrammetry and Remote Sensing, Vol. 62, No. 2, 78-92.

Kokaly, R. F. \& Clark, R. N., (1999). Spectroscopic Determination of Leaf Biochemistry Using Band-Depth Analysis of Absorption Features and Stepwise Multiple Linear Regression. Remote Sensing of Environment, Vol. 67, No. 3, 267-287.

Kruse, F. A., (2005). Multi-resolution segmentation for improved hyperspectral mapping. Proceedings, SPIE Symposium on Defense E Security, pp. 161, Orlando, FL. 
Kruse, F. A., et al., (1993). The Spectral Image-Processing System (SIPS) - Interactive Visualization and Analysis of Imaging Spectrometer Data. Remote Sensing of Environment, Vol. 44, No. 2-3, 145-163.

Lam, L. \& Suen, C. Y., (1994). A theoretical analysis of the application of majority voting topattern recognition. Proceedings of the 12th IAPR International Conference on Pattern Recognition and Computer Vision E Image Processing, pp. 418 - 420, Jerusalem.

Mandler, E. \& Schuermann, J., (1988). Combining the classification results of independent classifiers based on the Dempster/Shafer theory of evidence. Pattern Recognition and Artificial Intelligence, pp. 381-393, Amsterdam, Netherlands, Elsevier Science.

Nilsson, N. J., (1965). Learning machines, McGraw-Hill. New York.

Pal, M. \& Mather, P. M., (2003). An assessment of the effectiveness of decision tree methods for land cover classification. Remote Sensing of Environment, Vol. 86, No. 4, 554-565.

Richter, R., (1996). A spatially adaptive fast atmospheric correction algorithm. International Journal of Remote Sensing, Vol. 17, No. 6, 1201-1214.

Richter, R., (1998). Correction of satellite imagery over mountainous terrain. Applied Optics, Vol. 37, No. 18, 4004 - 4015.

Smith, G. M. \& Milton, E. J., (1999). The use of the empirical line method to calibrate remotely sensed data to reflectance. International Journal of Remote Sensing, Vol. 20, No. 13, 2653-2662.

Suen, C. Y. \& Lam, L., (2000). Multiple classifier combination methodologies for different output levels. Proceedings of First International Workshop on Multiple Classifier Systems (MCS 2000), pp. 52-66, Sardinia, Italy, Springer.

van der Meer, F. \& Bakker, W., (1997). Cross correlogram spectral matching: Application to surface mineralogical mapping by using AVIRIS data from Cuprite, Nevada. Remote Sensing of Environment, Vol. 61, No. 3, 371-382.

Vane, G. \& Goetz, A. F. H., (1988). Terrestrial imaging spectroscopy. Remote Sensing of Environment, Vol. 24, No. 1, 1-29.

Wen, X., et al., (2007a). Combining the Three Matched Filtering Methods in Mineral Information Extraction from Hyperspectral Data. Journal of China University of Geosciences, Vol. 18, No. Special Issue, 294-296.

Wen, X., et al., (2007b). A Simplified Method for Extracting Mineral Information From Hyperspectral Remote Sensing Image Using SAM Algorithm. 12th Conference of International Association for Mathematical Geology, Geomathematics and GIS Analysis of Resources, Environment and Hazards, pp. 526-529, Beijing, China.

Wen, X., et al., (2008a). CBERS-02 remote sensing data mining using decision tree algorithm. First International Workshop on Knowledge Discovery and Data Mining, pp. 86-89, Adelaide, Australia.

Wen, X., et al., (2008b). An investigation of the relationship between land cover ratio and urban heat island. 2008 International Congress on Image and Signal Processing, pp. 682686, Sanya, Hainan, China.

Winter, M. E., (1999). Fast autonomous spectral endmember determination in hyperspectral data. Proceedings of the Thirteenth International Conference on Applied Geologic Remote Sensing, pp. 337-344, Vancouver, British Columbia, Canada.

$\mathrm{Xu}$, L., et al., (1992). Methods of combining multiple classifiers and their applications to handwriting recognition. IEEE Transactions on Systems Man and Cybernetics, Vol. 22, No. 3, 418-435. 


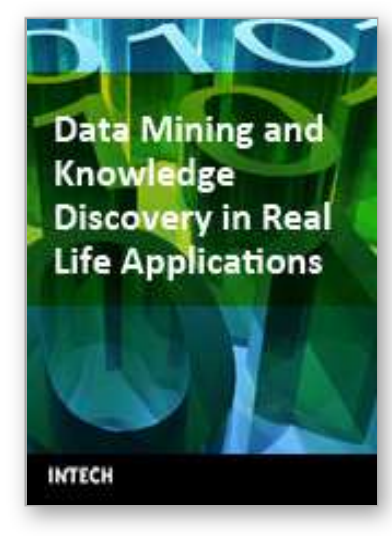

\section{Data Mining and Knowledge Discovery in Real Life Applications \\ Edited by Julio Ponce and Adem Karahoca}

ISBN 978-3-902613-53-0

Hard cover, 436 pages

Publisher I-Tech Education and Publishing

Published online 01, January, 2009

Published in print edition January, 2009

This book presents four different ways of theoretical and practical advances and applications of data mining in different promising areas like Industrialist, Biological, and Social. Twenty six chapters cover different special topics with proposed novel ideas. Each chapter gives an overview of the subjects and some of the chapters have cases with offered data mining solutions. We hope that this book will be a useful aid in showing a right way for the students, researchers and practitioners in their studies.

\section{How to reference}

In order to correctly reference this scholarly work, feel free to copy and paste the following:

Xing-Ping Wen, Xiao-Feng Yang and Guang-Dao Hu (2009). Hyperspectral Remote Sensing Data Mining Using Multiple Classifiers Combination, Data Mining and Knowledge Discovery in Real Life Applications, Julio Ponce and Adem Karahoca (Ed.), ISBN: 978-3-902613-53-0, InTech, Available from:

http://www.intechopen.com/books/data_mining_and_knowledge_discovery_in_real_life_applications/hyperspe ctral_remote_sensing_data_mining_using_multiple_classifiers_combination

\section{INTECH}

open science | open minds

\section{InTech Europe}

University Campus STeP Ri

Slavka Krautzeka 83/A

51000 Rijeka, Croatia

Phone: +385 (51) 770447

Fax: +385 (51) 686166

www.intechopen.com

\section{InTech China}

Unit 405, Office Block, Hotel Equatorial Shanghai

No.65, Yan An Road (West), Shanghai, 200040, China

中国上海市延安西路65号上海国际贵都大饭店办公楼 405 单元

Phone: +86-21-62489820

Fax: +86-21-62489821 
(C) 2009 The Author(s). Licensee IntechOpen. This chapter is distributed under the terms of the Creative Commons Attribution-NonCommercialShareAlike-3.0 License, which permits use, distribution and reproduction for non-commercial purposes, provided the original is properly cited and derivative works building on this content are distributed under the same license. 